\title{
THE PRODUCT OF NONPLANAR COMPLEXES \\ DOES NOT IMBED IN 4-SPACE
}

BY

BRIAN R. UMMEL

ABstract. We prove that if $K_{1}$ and $K_{2}$ are nonplanar simplicial complexes, then $K_{1} \times K_{2}$ does not imbed in $R^{4}$.

In this paper a proof is given of the following theorem:

THEOREM P. If $K_{1}$ and $K_{2}$ are finite simplicial complexes neither of which is homeomorphic to a subset of the euclidean plane $\mathbf{R}^{2}$, then their cartesian product $K_{1} \times K_{2}$ is not homeomorphic to any subset of euclidean 4-space $\mathbf{R}^{4}$.

This result answers a question originally posed by Professor Karl Menger in [5]. I wish to thank Professor Joseph Zaks for showing me this problem.

1. Preliminaries. We say a space $X$ imbeds in euclidean $n$-space $\mathbf{R}^{n}$ if there is an imbedding (i.e., homeomorphism into) $f: X \rightarrow \mathbf{R}^{n}$. If $X$ imbeds in $\mathbf{R}^{2}$ we say that $X$ is planar. For a proof of the following see [4]:

Proposition 1.1. If $K$ is a finite nonplanar simplicial complex then $K$ contains a subspace homeomorphic to one of the following spaces:

a. $K_{5}^{1}$, the complete graph on 5 vertices (or, if you prefer, the 1-skeleton of a 4-simplex);

b. $K_{3,3}^{1}$, the join of 2 3-point sets;

c. $S^{2}$, the 2-sphere; or

d. $Q^{2}=\left\{x \in \mathbf{R}^{3}: x_{3}=0\right.$ and $x_{1}^{2}+x_{2}^{2} \leqslant 1$ or $x_{1}=x_{2}=0$ and $0 \leqslant x_{3} \leqslant$ 1\}.

We will henceforth assume that the complexes $K_{1}$ and $K_{2}$ of Theorem $\mathrm{P}$ are chosen from the list of Proposition 1.1, and that we have chosen imbeddings

$$
f_{1}: K_{1} \rightarrow \mathbf{R}^{3} \text { and } f_{2}: K_{2} \rightarrow \mathbf{R}^{3} \text {. }
$$

To clarify notations we recall some standard definitions. Let $\pi=\{1, \tau\}$ be the multiplicative group of order 2. A $\pi$-space $X$ is a Hausdorff space together with a fixed point free involution $\tau: X \rightarrow X$; this involution defines a free

Received by the editors May 10, 1977.

AMS (MOS) subject classifications (1970). Primary 55A20, 57C35.

Key words and phrases. Imbedding, imbedding class, minimal complex, low-dimensional complex.

C American Mathematical Society 1978 
$\pi$-action on $X$ and we denote the orbit space of this action by $X / \pi$. The natural projection $p: X \rightarrow X / \pi$ is then a 2-fold covering. If $X$ and $Y$ are $\pi$-spaces then a map $f: X \rightarrow Y$ is $\pi$-equivariant if $f \cdot \tau=\tau \cdot f$. Homotopies are equivariant if they are $\pi$-equivariant at each stage. If $K$ is a Hausdorff space, the deleted product of $K$ is

$$
D_{2} K=\{(x, y) \in K \times K: x \neq y\}
$$

Using the action $\tau(x, y)=(y, x), D_{2} K$ is a $\pi$-space and we denote the orbit space by $\Sigma_{2} K$. Let $S^{\infty}=$ proj $\lim S^{n}$ under the natural inclusions and $\tau$ : $S^{\infty} \rightarrow S^{\infty}$ be the limit of the antipodal maps; set $P^{\infty}=S^{\infty} / \tau$. If $K$ is paracompact there is a $\pi$-equivariant map $\hat{k}: D_{2} K \rightarrow S^{\infty}$ and any two such maps are $\pi$-equivariantly homotopic (cf. [2]). Using the induced map $k$ : $\Sigma_{2} K \rightarrow P^{\infty}$ and singular cohomology with $Z_{2}$ coefficients we define the $n t h$ mod-2 imbedding class of $K$ by

$$
\Phi_{2}^{n}(K)=K^{*}\left(w^{n}\right) \in H^{n}\left(\Sigma_{2} K ; Z_{2}\right)
$$

where $w^{n}$ is the nonzero element of $H^{n}\left(P^{\infty} ; Z_{2}\right), n \geqslant 0$. The following is an immediate consequence of the definition.

Proposition 1.2. a. If $K$ and $L$ are paracompact, $f: K \rightarrow L$ is an imbedding, and $F: \Sigma_{2} K \rightarrow \Sigma_{2} L$ is the induced map, then $F^{*}\left(\Phi_{2}^{n}(L)\right)=\Phi_{2}^{n}(K)$. b. For $n>0, D_{2} \mathbf{R}^{n}$ is $\pi$-equivariantly homotopy equivalent to $S^{n-1}$ (with antipodal action); thus $\Phi_{2}^{i}\left(\mathbf{R}^{n}\right) \neq 0$ iff $0 \leqslant i \leqslant n-1$.

Thus $\Phi_{2}^{n}(K)=0$ is a necessary condition for a paracompact space to imbed in $\mathbf{R}^{n}$. In $\S 3$ we prove Theorem $\mathrm{P}$ by showing that $\Phi_{2}^{4}\left(K_{1} \times K_{2}\right) \neq 0$. The information we need about the deleted products of $K_{1}$ and $K_{2}$ is summarized in the following:

Proposition 1.3. If $K$ is one of the four complexes $K_{5}^{1}, K_{3,3}^{1}, S^{2}$ or $Q^{2}$ of Proposition 1.1, then

a. $\Phi_{2}^{2}(K) \neq 0$;

b. $D_{2} K$ is $\pi$-equivariantly homotopy equivalent to a closed 2-manifold of genus $g$, where $g=6$ if $K=K_{5}^{1}, g=4$ if $K=K_{3,3}^{1}$ and $g=0$ if $K=S^{2}$ or $Q^{2}$;

c. if $f: K \rightarrow \mathbf{R}^{3}$ is an imbedding and $\hat{F}: D_{2} K \rightarrow D_{2} \mathbf{R}^{3}$ is the induced map, then $\hat{F}^{*}: H^{2}\left(D_{2} \mathbf{R}^{3}\right) \rightarrow H^{2}\left(D_{2} K\right)$ is an isomorphism.

Proof. For a and b see [7] and [8]. For $\mathrm{c}$ we have the following commutative diagram whose rows are exact Gysin sequences (where we interpret a 2-fold covering as a 0 -sphere bundle cf. [6]):

$$
\begin{array}{ccccccc}
\rightarrow & H^{2}\left(\Sigma_{2} \mathbf{R}^{3}\right) & \stackrel{p^{\prime *}}{\rightarrow} & H^{2}\left(D_{2} \mathbf{R}^{3}\right) & \stackrel{\rho^{\prime}}{\rightarrow} & H^{2}\left(\Sigma_{2} \mathbf{R}^{3}\right) & \rightarrow 0 \\
& \downarrow F^{*} & & & \downarrow \hat{F}^{*} & & \\
& H^{2}\left(\Sigma_{2} K\right) & \stackrel{p^{*}}{\rightarrow} & H^{2}\left(D_{2} K\right) & \stackrel{\rho}{\rightarrow} & H^{2}\left(\Sigma_{2} K\right) & \rightarrow 0
\end{array}
$$


where $F: \Sigma_{2} K \rightarrow \Sigma_{2} \mathbf{R}^{3}$ is the map induced by $\hat{F}$. All six groups in the diagram are isomorphic to $Z_{2}$ and so $\rho$ and $\rho^{\prime}$ are isomorphisms. Thus $\hat{F}^{*}$ is an isomorphism.

Using $\left[g_{1}, \ldots, g_{n}\right]$ to denote the $Z_{2}$-module with basis $\left\{g_{1}, \ldots, g_{n}\right\}$ or the zero module if $n=0$, we can write

$$
\begin{array}{ll}
H^{0}\left(K_{1}\right)=\left[\omega^{0}\right], & H^{0}\left(K_{2}\right)=\left[\mu^{0}\right], \\
H^{1}\left(K_{1}\right)=\left[\omega^{1}, \ldots, \omega_{\eta}^{1}\right], & H^{1}\left(K_{2}\right)=\left[\mu_{1}^{1}, \ldots, \mu_{\sigma}^{1}\right],
\end{array}
$$

where $\eta$ or $\sigma\}$ is $0,0,4$, or 6 depending upon whether $K_{1}$ \{or $\left.K_{2}\right\}$ is $S^{2}, Q^{2}$, $K_{3,3}^{1}$, or $K_{5}^{1}$. Here the superscripts denote dimension rather than exponents. If $K_{1}\left\{K_{2}\right\}$ is $S^{2}$ or $Q^{2}$ we denote $H^{2}\left(K_{1}\right)=\left[\omega^{2}\right]\left\{H^{2}\left(K_{2}\right)=\left[\mu^{2}\right]\right\}$; otherwise $H^{2}\left(K_{1}\right)=0\left\{H^{2}\left(K_{2}\right)=0\right\}$. We also need to assume that if $\eta \neq 0\{\sigma \neq 0\}$ then the above basis for $H^{1}\left(K_{1}\right)\left\{H^{1}\left(K_{2}\right)\right\}$ is dual to a basis which satisfies the following:

LEMMA 1.4. If $K$ is a finite 1-dimensional simplicial complex and $i: D_{2} K \rightarrow K$ $\times K$ is the inclusion map, then there is a basis $\left\{\beta_{1}, \ldots, \beta_{m}\right\}$ for $H_{1}(K)$ such that if $\beta \in H_{2}\left(D_{2} K\right)$ then $i_{*}(\beta)=\Sigma c_{i j}\left(\beta_{i} \times \beta_{j}\right)$ where $c_{i j} \in Z_{2}, c_{i i}=0$ for $i=1, \ldots, m$ and " $\times$ " denotes cross product.

Proof. Let $D_{2}^{P}(K)=\left\{\left(x_{1}, x_{2}\right) \in K \times K: c\left(x_{1}\right) \cap c\left(x_{2}\right)=\varnothing\right\}$ where $c\left(x_{j}\right)$ is the smallest closed simplex of $K$ containing $x_{j}$. Then, by [9], $D_{2}^{P}(K)$ is a strong $\pi$-equivariant deformation retract of $D_{2} K$, and so we can use the inclusion $j: D_{2}^{P} K \rightarrow K \times K$ instead of $i$. Let $\left\{\sigma_{1}, \ldots, \sigma_{m+n}\right\}$ be the 1-simplices of $K$ numbered so that $\left\{\sigma_{m+1}, \ldots, \sigma_{m+n}\right\}$ form a maximal tree $\Gamma$ of $K$. We also use $\sigma_{i}$ to denote the linear singular 1-simplex whose image is $\sigma_{i}$; there is no orientation problem since we are using $Z_{2}$ coefficients. For $i=$ $1, \ldots, m$ set $\beta_{i}=\left[\sigma_{i}+\lambda_{i}\right] \in H_{1}\left(K_{1}\right)$ where $\lambda_{i}$ is a sum of simplices of $\Gamma$. For $i>m$ we set $\lambda_{i}=\sigma_{i}$. Suppose $\beta \in H_{2}\left(D_{2}^{p} K\right)$. Then $\beta=\left[\Sigma k_{i j}\left(\sigma_{i} \times \sigma_{j}\right)\right]$ where $k_{i j} \in Z_{2}$ and $k_{i i}=0$ for all $i$. We have

$$
\begin{aligned}
\sigma_{i} \times \sigma_{j} & =\left(\sigma_{i}+\lambda_{i}-\lambda_{i}\right) \times\left(\sigma_{j}+\lambda_{j}-\lambda_{j}\right) \\
& =\left(\sigma_{i} \times \lambda_{i}\right) \times\left(\sigma_{j} \times \lambda_{j}\right)-\lambda_{i} \times \sigma_{j}-\sigma_{i} \times \lambda+\lambda_{i}+\lambda_{j} .
\end{aligned}
$$

So if $i \neq j, \sigma_{i} \times \sigma_{j}=\left(\sigma_{i}+\lambda_{j}\right)+\gamma_{i j}$ where $\gamma_{i j}$ is a 2-chain of $X \times \Gamma \cup \Gamma \times X$. So $\beta=\left[\Sigma k_{i j}\left(\sigma_{i}+\lambda_{i}\right) \times\left(\sigma_{j}+\lambda_{j}\right)+\gamma\right]$ where $\gamma$ is a 2-chain of $\Gamma \times X \cup X \times$ $\Gamma$. In fact $\gamma$ is a 2-cycle of $\Gamma \times X \cup X \times \Gamma$ and since $H_{2}(\Gamma \times X \cup X \times \Gamma)$ $=0, j_{*}(\gamma)=0$. Thus $j^{*}(\beta)=\Sigma k_{i j}\left(\beta_{i} \times \beta_{j}\right)$. Thus $\left\{\beta_{1}, \ldots, \beta_{n}\right\}$ is the desired basis. This proves Lemma 1.4.

LEMMA 1.5. If $K=K_{5}^{1}, K_{3,3}^{1}, S^{2}$ or $Q^{2}$, then the inclusion map $J: D_{2} K \rightarrow K$ $\times K$ induces an isomorphism $j^{*}: H^{1}(K \times K) \rightarrow H^{1}\left(D_{2} K\right)$.

Proof. For $K=S^{2}$ or $Q^{2}$ both groups are trivial. If $K=K_{5}^{1}$ or $K_{3,3}^{1}$ then 
by [8] $D_{2}(C K)$ is $\pi$-equivariantly homotopy equivalent to $S^{3}$. So in the exact sequence (cf. [1])

$$
\rightarrow H^{n}\left(D_{2} C K\right) \rightarrow H^{n}(K) \oplus H^{n}(K) \stackrel{\alpha}{\rightarrow} H^{n}\left(D_{2} K\right) \rightarrow H^{n+1}\left(D_{2} C K\right) \rightarrow
$$

where $\alpha(u, v)=q_{1}^{*}(u)+q_{2}^{*}(v), q_{i}: D_{2} K \rightarrow K$ given by $q_{i}\left(x_{1}, x_{2}\right)=x_{i}$, we have $\alpha$ is an isomorphism if $n=1$. Using this and the Künneth Theorem proves Lemma 1.5.

Using Lemma 1.5, Proposition 1.5 and the above bases we have:

$$
\begin{aligned}
& H^{0}\left(D_{2} K_{1}\right)=\left[\omega^{0} \times \omega^{0}\right], H^{1}\left(D_{2} K_{1}\right)=\left[\omega^{0} \times \omega_{i}^{1}, \omega_{i}^{1} \times \omega^{0} ; i=1, \ldots, \eta\right], \\
& H^{2}\left(D_{2} K_{1}\right)=\left[\Omega^{2}\right],
\end{aligned}
$$

and

$$
\begin{aligned}
H^{0}\left(D_{2} K_{2}\right)=\left[\mu^{0} \times \mu^{0}\right], \quad H^{1}\left(D_{2} K_{2}\right)=\left[\mu^{0} \times \mu_{i}^{1}, \mu_{i}^{1} \times \mu^{0} ; i=1, \ldots, \sigma\right], \\
H^{2}\left(D_{2} K_{2}\right)=\left[\Lambda^{2}\right]
\end{aligned}
$$

where " $X$ " denotes cross product followed by restriction.

For Hausdorff spaces $K$ and $L$ we define

$$
\begin{aligned}
\hat{J}_{0}(K, L)= & D_{2} K \times D_{2} L, \quad \hat{J}_{1}(K, L)=D_{2} K \times(L \times L), \\
& \hat{J}_{2}(K, L)=(K \times K) \times D_{2} L .
\end{aligned}
$$

Using $\tau\left(x_{1}, x_{2}, y_{1}, y_{2}\right)=\left(x_{2}, x_{1}, y_{2}, y_{1}\right), \hat{J}_{k}(K, L)$ becomes a $\pi$-space and we denote the quotient spaces by $J_{k}(K, L)$ for $k=0,1,2$.

LEMMA 1.6. If $K$ and $L$ are Hausdorff spaces then $D_{2}(K \times L)$ is $\pi$-equivariantly homeomorphic to $\hat{J}_{1}(K, L) \cup \hat{J}_{2}(K, L)$. Moreover, $\left\{J_{1}(K, L)\right.$, $\left.J_{2}(K, L)\right\}$ is an excisive couple and $J_{1}(K, L) \cap J_{2}(K, L)=J_{0}(K, L)$.

Proof. Clearly $\hat{\varphi}: D_{2}(K \times L) \rightarrow \hat{J}_{1}(K, L) \cup \hat{J}_{2}(K, L)$ defined by

$$
\hat{\varphi}\left(x_{1}, y_{1}, x_{2}, y_{2}\right)=\left(x_{1}, x_{2}, y_{1}, y_{2}\right)
$$

is a $\pi$-equivariant homeomorphism. Since $J_{1}(K, L)$ and $J_{2}(K, L)$ are open in their union, the couple is excisive.

For $k=0,1$, and 2 let

$$
\begin{array}{ll}
\hat{J}_{k}=\hat{J}_{k}\left(K_{1}, K_{2}\right), & \hat{J}_{k}^{\prime}=\hat{J}_{k}\left(\mathbf{R}^{3} ; \mathbf{R}^{3}\right), \\
J_{k}=J_{k}\left(K_{1}, K_{2}\right), & J_{k}^{\prime}=J_{k}\left(\mathbf{R}^{3} ; \mathbf{R}^{3}\right),
\end{array}
$$

and $\hat{F}_{k}: \hat{J}_{k} \rightarrow \hat{J}_{k}, F_{k}: J_{k} \rightarrow J_{k}^{\prime}$ denote the maps induced by the imbeddings $f_{j}$ : $K_{j} \rightarrow \mathbf{R}^{3}, j=1,2$. We also have maps

$$
\hat{F}: D_{2}\left(K_{1} \times K_{2}\right) \rightarrow D_{2}\left(\mathbf{R}^{3} \times \mathbf{R}^{3}\right)
$$

and

$$
F: \Sigma_{2}\left(K_{1} \times K_{2}\right) \rightarrow \Sigma_{2}\left(\mathbf{R}^{3} \times \mathbf{R}^{3}\right)
$$


Finally let $\hat{i}_{k}: \hat{J}_{0} \rightarrow \hat{J}_{k}$ and $i_{k}: J_{0} \rightarrow J_{k}$ be the inclusions for $k=1,2$ and $p_{k}$ : $\hat{J}_{k} \rightarrow J_{k}, p_{k}^{\prime}: \hat{J}_{k}^{\prime} \rightarrow J_{k}^{\prime}$ be the natural projections for $j=0,1,2$.

LEMMA 1.7. $F_{0}^{*}: H^{4}\left(J_{0}^{\prime}\right) \rightarrow H^{4}\left(J_{0}\right)$ is an isomorphism.

Proof. $\hat{J}_{0}^{\prime}$ and $\hat{J}_{0}$ are $\pi$-equivariantly homotopy equivalent to closed $4-$ manifolds; hence $J_{0}$ and $J_{0}^{\prime}$ are homotopy equivalent to closed 4-manifolds. Thus in the commutative diagram

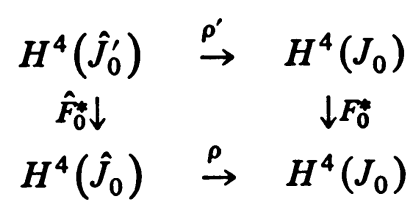

where $\rho^{\prime}$ and $\rho$ are from the appropriate Gysin sequences, and Proposition 1.3.c, $F_{0}^{*}$ is an isomorphism. This proves Lemma 1.7.

2. The spectral sequence of a double covering. The proof of Theorem $P$ requires the following in which we use the notation of $\$ 1$.

LEMMA 2.1. $\operatorname{Ker}\left[p_{1}^{*}: H^{4}\left(J_{1}\right) \rightarrow H^{4}\left(\hat{J}_{1}\right)\right] \subseteq \operatorname{Im}\left[i_{1}^{*}: H^{4}\left(J_{1}\right) \rightarrow H^{4}\left(J_{0}\right)\right]$.

The proof of Lemma 2.1 requires using the cohomology spectral sequence of a covering (cf. [3]) specialized to the case of a double covering which allows explicit identification of the $E_{1}$-term and the $E_{1}$ differential operators. The properties of this spectral sequence are summarized in the following:

Proposition 2.2. If $X$ is a $\pi$-space, there is a natural first quadrant $E_{1}$-spectral sequence $\left\{E_{r}^{p, q}(X), d_{r}^{p, q}\right\}_{r=1}^{\infty}$ convergent to $H^{*}\left(X / \pi, Z_{2}\right)$ with the following properties:

a. $E_{1}^{p, q}(X)=H^{q}\left(X ; Z_{2}\right)$,

b. $d_{1}^{p, q}: E_{1}^{p, q}(X) \rightarrow E_{1}^{p+1, q}(X)$ is given by $d_{1}^{p, q}(\alpha)=\alpha+\tau \alpha$ where $\tau$ : $H^{1}\left(H ; Z_{2}\right)=H^{1}\left(X, Z_{2}\right)$ is the homomorphism induced by the involution $\tau$ : $X \rightarrow X$.

c. For each $n$ there is a natural decreasing filtration $\left\{F_{p} H^{n}(X / n)\right\}_{p=0}^{\infty}$ of $H^{n}\left(X_{\pi}, Z_{2}\right)$ such that $F_{0} H^{n}(X / \pi)=H^{n}\left(X / \pi ; Z_{2}\right), F_{n+1} H^{n}(X / \pi)=0$ and for each $p \geqslant 0$ there is a natural short exact sequence

$$
0 \rightarrow F_{p+1} H_{n}(X / \pi) \rightarrow F_{p} H_{n}(X / \pi) \stackrel{q}{\rightarrow} E_{\infty}^{p, n-p}(X) \rightarrow 0 .
$$

d. The projection induced map $p^{*}: H^{n}\left(X / \pi ; Z_{2}\right) \rightarrow H^{n}\left(X ; Z_{2}\right)$ is the composition:

$$
H^{n}(X / \pi)=F_{0} H^{n}(X / \pi) \stackrel{q}{\rightarrow} E_{\infty}^{0, n}(X) \subseteq E_{1}^{0, n}(X)=H^{\prime}(X) .
$$

For the remainder of this section and the next we assume all coefficients to be $Z_{2}$ and suppress this in the notation. 
LEMMA 2.3. If $p \geqslant 1$ then $d_{3}^{p, 2}: E_{3}^{p, 2}\left(D_{2} K_{1}\right) \rightarrow E_{3}^{p+3,0}\left(D_{2} K_{1}\right)$ is an isomorphism with $E_{3}^{p, 2}\left(D_{2} K_{1}\right)=\left[\Omega^{2}\right]$.

Proof. Using the calculations of $\S 1$ and Proposition 2.2.b,

$$
\begin{aligned}
& E^{p, 0}\left(D_{2} K_{1}\right)=\left[\omega^{0} \times \omega^{0}\right], p \geqslant 0, E_{2}^{p, 1}\left(D_{2} K_{1}\right)=0, p \geqslant 1, \\
& E_{2}^{p, 2}\left(D_{2} K_{1}\right)=\left[\Omega^{2}\right], p \geqslant 0 .
\end{aligned}
$$

Thus $E_{3}^{p, 0}\left(D_{2} K_{1}\right)=E_{2}^{p, 0}\left(D_{2} K_{1}\right)=\left[\omega^{0} \times \omega^{0}\right]$ if $p \geqslant 3$ and $E_{3}^{p, 2}\left(D_{2} K_{1}\right)=$ $E_{2}^{p, 2}\left(D_{2} K_{1}\right)=\left[\Omega^{2}\right]$ if $p \geqslant 0$. Since $H^{n}\left(\Sigma_{2} K_{1}\right)=0$ if $n>2$,

$$
d_{3}^{p, 2}: E_{3}^{p, 2}\left(D_{2} K_{1}\right) \rightarrow E_{3}^{p+3,0}\left(D_{2} K_{1}\right)
$$

must be an isomorphism. This proves Lemma 2.3.

LEMMA 2.4. $E_{\infty}^{p, 4-p}\left(\hat{J}_{0}\right)=0$ if $p \geqslant 3$.

Proof. Using the calculations of $\S 1$ and the Künneth formula,

$$
\begin{aligned}
H^{0}\left(\hat{J}_{0}\right)= & {\left[\omega^{0} \times \omega^{0}\right] \otimes\left[\mu^{0} \times \mu^{0}\right], } \\
H^{1}\left(\hat{J}_{0}\right)= & {\left[\omega^{0} \times \omega^{0}\right] \otimes\left[\mu_{i}^{1} \times \mu^{0}, \mu^{0} \times \mu_{i}^{1} ; i=1, \ldots, \sigma\right] } \\
& \oplus\left[\omega_{i}^{1} \times \omega^{0}, \omega^{0} \times \omega_{i}^{1} ; i=1, \ldots, \eta\right] \otimes\left[\mu^{0} \times \mu^{0}\right],
\end{aligned}
$$

where $\eta$ and $\sigma$ are the ranks of $H^{1}\left(K_{1}\right)$ and $H^{1}\left(K_{2}\right)$ respectively. Using the diagonal $\pi$-action and Proposition 2.2.b

$$
E_{2}^{4,0}\left(\hat{J}_{0}\right)=\left[\omega^{0} \times \omega^{0}\right] \otimes\left[\mu^{0} \times \mu^{0}\right], \quad E_{2}^{p, 1}\left(\hat{J}_{0}\right)=0 \quad \text { if } p>0 .
$$

Since $E_{2}^{2,1}\left(\hat{J}_{0}\right)=0, E_{3}^{4,0}\left(\hat{J}_{0}\right)=E_{2}^{4,0}\left(\hat{J}_{0}\right)$ and

$$
\pi_{1}^{*}: E_{3}^{4,0}\left(D_{2} K_{1}\right) \rightarrow E_{3}^{4,0}\left(\hat{J}_{0}\right)
$$

is an isomorphism where $\pi_{1}: \hat{J}_{0} \rightarrow D_{2} K_{1}$ is the projection. From the commutative diagram

$$
\begin{array}{ccc}
E_{3}^{1,2}\left(D_{2} K_{1}\right) & \stackrel{\pi^{*}}{\rightarrow} & E_{3}^{1,2}\left(\hat{J}_{0}\right) \\
d_{3}^{1,2} \downarrow & & \downarrow d_{3}^{1,2} \\
E_{3}^{4,0}\left(D_{2} K_{1}\right) & \stackrel{\pi_{l}^{*}}{\rightarrow} & E_{3}^{4,0}\left(\hat{J}_{0}\right)
\end{array}
$$

and Lemma $2.3, d_{3}^{1,2}: E_{3}^{1,2}\left(\hat{J}_{0}\right) \rightarrow E_{3}^{4,0}\left(\hat{J}_{0}\right)$ is surjective. Thus $E_{\infty}^{4,0}\left(\hat{J}_{0}\right)=$ $E_{4}^{4,0}\left(\hat{J}_{0}\right)=E_{4}^{4,0}\left(\hat{J}_{0}\right)=0$. From above, $E_{\infty}^{3,1}\left(\hat{J}_{0}\right)=0$. This proves Lemma 2.4 .

LEMMA 2.5. $E_{\infty}^{1,3}\left(\hat{J}_{1}\right)=0$ and $\hat{1}^{*}: E_{\infty}^{2,2}\left(\hat{J}_{0}\right)$ is the zero homomorphism.

Proof. We first compute $E_{2}^{p, q}\left(\hat{J}_{1}\right)$ in 3-cases. If $K_{2}=K_{5}^{1}$ or $K_{3,3}^{1}$, then 


$$
\begin{aligned}
H^{1}\left(\hat{J}_{1}\right)=\left[\omega^{0} \times \omega^{0}\right] \otimes\left[\mu^{0} \times \mu_{i}^{1}, \mu_{i}^{1} \times \mu_{0} ; i=1, \ldots, \sigma\right] \\
\oplus\left[\omega^{0} \times \omega_{i}^{1}, \omega_{i}^{1} \times \omega^{0} ; i=1, \ldots, \eta\right] \otimes\left[\mu^{0} \times \mu^{0}\right], \\
H^{2}\left(\hat{J}_{1}\right)=\left[\omega^{0} \times \omega^{0}\right] \otimes\left[\mu_{i}^{1} \times \mu_{j}^{1} ; i, j=1, \ldots, \sigma\right] \\
\\
\oplus\left[\omega^{0} \times \omega_{i}^{1}, \omega_{i}^{1} \times \omega^{0} ; i=1, \ldots, \eta\right] \\
\otimes\left[\mu^{0} \times \mu_{i}^{1}, \mu_{i}^{1} \times \mu^{0} ; i=1, \ldots, \sigma\right] \\
\oplus\left[\Omega^{2}\right] \otimes\left[\mu^{0} \times \mu^{0}\right] .
\end{aligned}
$$

Using the diagonal $\pi$-action on $\hat{J}_{1}$ and Proposition 2.2

$$
\begin{aligned}
& E_{2}^{p, 1}\left(\hat{J}_{1}\right)=0 \text { if } p>0, \\
& E_{2}^{2,2}\left(\hat{J}_{1}\right)=\left[\omega^{0} \times \omega^{0}\right] \otimes\left[\mu_{i}^{1} \times \mu_{i}^{1} ; i=1, \ldots, \sigma\right] \oplus\left[\Omega^{2}\right] \otimes\left[\mu^{0} \times \mu^{0}\right], \\
& E_{2}^{1,3}\left(\hat{J}_{1}\right)=0 .
\end{aligned}
$$

If $K_{2}=S^{2}$ and we set $H_{2}\left(K_{2}\right)=\left[\mu^{2}\right]$ then

$$
\begin{aligned}
& H^{1}\left(\hat{J}_{1}\right)=\left[\omega^{0} \times \omega_{i}^{1}, \omega_{i}^{1} \times \omega^{0} ; i=1, \ldots, \eta\right] \otimes\left[\mu^{0} \times \mu^{0}\right], \\
& H^{2}\left(\hat{J}_{1}\right)=\left[\omega^{0} \times \omega^{0}\right] \otimes\left[\mu^{0} \times \mu^{2}, \mu^{2} \times \mu^{0}\right] \oplus\left[\Omega^{2}\right] \otimes\left[\mu^{0} \times \mu^{0}\right] .
\end{aligned}
$$

Therefore

$$
\begin{gathered}
E_{2}^{p, 1}\left(\hat{J}_{1}\right)=0 \text { if } p>0, \quad E_{2}^{2,2}\left(\hat{J}_{1}\right)=\left[\Omega^{2} \times \mu^{0} \times \mu^{0}\right], \\
E_{2}^{1,3}\left(\hat{J}_{1}\right)=0 .
\end{gathered}
$$

Finally if $K_{2}=Q_{2}$, then

$$
\begin{gathered}
E_{2}^{p, 1}\left(\hat{J}_{1}\right)=0 \text { if } p>0, \quad E_{2}^{2,2}\left(\hat{J}_{1}\right)=H^{2}\left(\hat{J}_{1}\right)=\left[\Omega^{2} \times \mu^{0} \times \mu^{0}\right], \\
E_{2}^{1,3}\left(\hat{J}_{1}\right)=H^{3}\left(\hat{J}_{1}\right)=0 .
\end{gathered}
$$

In all cases $E_{2}^{p, 0}\left(\hat{J}_{1}\right)=\left[\omega^{0} \times \omega^{0} \times \mu^{0} \times \mu^{0}\right]$. Since $\pi_{1}^{*}: H^{1}\left(D_{2} K_{1}\right) \rightarrow H^{2}\left(\hat{J}_{1}\right)$ is an isomorphism, where $\pi_{1}: \hat{J}_{1} \rightarrow D_{2} K_{1}$ is the projection, and $E_{2}^{p, 1}\left(\hat{J}_{1}\right)=0$ if $p>0$, we have

$$
\pi_{1}^{*}: E_{3}^{p, 0}\left(D_{2} K_{1}\right) \rightarrow E_{3}^{p, 0}\left(\hat{J}_{1}\right)
$$

is an isomorphism for $p>2$. Consider the commutative diagram

$$
\begin{array}{ccc}
E_{3}^{2,2}\left(D_{2} K_{1}\right) & \stackrel{\pi_{1}^{*}}{\rightarrow} & E_{3}^{2,2}\left(\hat{J}_{1}\right) \\
d_{3}^{2,2} \downarrow & & \downarrow d_{3}^{2,2} \\
E_{3}^{5,0}\left(D_{2} K_{1}\right) & \stackrel{\pi_{1}^{*}}{\rightarrow} & E_{3}^{5,0}\left(\hat{J}_{1}\right)
\end{array}
$$

Using Lemma 2.3, and $\pi_{1}^{*}\left(\left[\Omega^{2}\right]\right)=\left[\Omega^{2} \times \omega^{0} \times \omega^{0}\right]$ we have

$$
d_{3}^{2,2}\left(\left[\Omega^{2} \times \omega^{0} \times \omega^{0}\right]\right) \neq 0 .
$$


Therefore

$$
E_{4}^{2,2}\left(\hat{J}_{1}\right)=\left\{\begin{array}{l}
{\left[\omega^{0} \times \omega^{0} \times \mu_{i}^{1} \times \mu_{i}^{1} ; i=1, \ldots, \sigma\right] \text { if } K_{2}=K_{5}^{\prime} \text { or } K_{3,3}^{\prime},} \\
0 \text { if } K_{2}=S^{2} \text { or } Q^{2} .
\end{array}\right.
$$

To show that $\hat{1}^{*}\left(\left[\omega^{0} \times \omega^{0} \times \mu_{i}^{1} \times \mu_{i}^{1}\right]\right)=0$, let $\left\{\alpha_{1}^{1}, \ldots, \alpha_{\sigma}^{1}\right\}$ be the basis of $H_{1}\left(K_{2}\right)$ dual to $\left\{\mu_{1}^{1}, \ldots, \mu_{\sigma}^{1}\right\}$ as in Lemma 1.4 and let $\alpha^{2}$ denote the nonzero element of $\mathrm{H}_{2}\left(D_{2} K_{2}\right)$. If $j: D_{2} K_{2} \rightarrow K_{2} \times K_{2}$ is the inclusion, then by Lemma 1.4

$$
\begin{aligned}
\left\langle j^{*}\left(\mu_{i}^{1} \times \mu_{i}^{1}\right), \alpha^{2}\right\rangle & =\left\langle\mu_{i}^{1} \times \mu_{i}^{1}, j_{*}\left(\alpha^{2}\right)\right\rangle=\left\langle\mu_{i}^{1} \times \mu_{i}^{1}, \sum_{j \neq k} c_{j k}\left(\alpha_{j}^{1} \times \alpha_{k}^{1}\right)\right\rangle \\
& =\sum_{j \neq k} c_{j k}\left\langle\mu_{i}^{1}, \alpha_{j}^{1}\right\rangle\left\langle\mu_{i}^{1}, \alpha_{k}^{1}\right\rangle=\sum_{j \neq k} c_{j k} \delta_{j k}=0 .
\end{aligned}
$$

Thus $j^{*}\left(\mu_{i}^{1} \times \mu_{i}^{1}\right)=0$. So $\hat{\imath}^{*}\left(\left[\omega^{0} \times \omega^{0} \times \mu_{i}^{1} \times \mu_{i}^{1}\right]\right)=0$. This proves Lemma 2.5.

Proof of Lemma 2.1. By Proposition 2.2.d, if $p_{1}: \hat{J}_{1} \rightarrow J_{1}$ is the natural projection then $p_{1}^{*}: H^{4}\left(J_{1}\right) \rightarrow H^{4}\left(\hat{J}_{1}\right)$ is the composition

$$
H^{4}\left(J_{1}\right)=F^{0} H^{4}\left(J^{\prime}\right) \stackrel{q}{\rightarrow} E_{\infty}^{0,4}\left(\hat{J}_{1}\right) \subseteq E_{1}^{0,4}\left(\hat{J}_{1}\right)=H^{4}\left(J_{1}\right) .
$$

So using Proposition 2.2.c we have

$$
\begin{aligned}
& \operatorname{ker}\left[p_{1}^{*}: H^{4}\left(J_{1}\right) \rightarrow H^{4}\left(\hat{J}_{1}\right)\right] \\
& \quad=\operatorname{ker}\left[q: F^{0} H^{4}\left(J_{1}\right) \rightarrow E_{\alpha}^{0,4}\left(\hat{J}_{1}\right)\right]=F_{1} H^{4}\left(J_{1}\right) .
\end{aligned}
$$

By Lemma 2.5, $E_{\infty}^{1,3}\left(\hat{J}_{1}\right)=0$; thus $F_{2} H^{4}\left(J_{1}\right)=F_{1} H^{4}\left(J_{1}\right)$. Thus $i_{1}^{*} \mid\left(\operatorname{ker} p_{1}^{*}\right)=0$ if and only if $i_{1}^{*} \mid F_{2} H^{4}\left(J_{1}\right)=0$. Now consider the following commutative diagram with exact rows:

$$
\begin{aligned}
& 0 \rightarrow F_{3} H^{4}\left(J_{1}\right) \rightarrow F_{2} H^{4}\left(J_{1}\right) \rightarrow E_{\infty}^{2,2}\left(\hat{J}_{1}\right) \rightarrow 0 \\
& \downarrow i_{1}^{*} \quad \downarrow i^{*} \quad \downarrow i_{i}^{*} \\
& 0 \rightarrow F_{3} H^{4}\left(J_{0}\right) \rightarrow F_{2} H^{4}\left(J_{0}\right) \rightarrow E_{\infty}^{2,2}\left(\hat{J}_{0}\right) \rightarrow 0
\end{aligned}
$$

By Lemma 2.4, $E_{\infty}^{4,1}\left(\hat{J}_{0}\right) \cong 0$; thus $F_{3} H^{4}\left(J_{0}\right)=0$ and so the projection

$$
F_{2} H^{4}\left(J_{0}\right) \rightarrow E_{\infty}^{2,2}\left(\hat{J}_{0}\right)
$$

is an isomorphism. But by Lemma $2.5, \hat{\imath}_{1}^{*}: E_{\infty}^{2,2}\left(\hat{J}_{1}\right) \rightarrow E_{\infty}^{2,2}\left(\hat{J}_{0}\right)$ is zero. Thus $i_{1}^{*}: F_{2} H^{4}\left(J_{1}\right) \rightarrow F_{2} H^{4}\left(J_{0}\right)$ is zero. This proves Lemma 2.1 .

3. Proof of Theorem $P$. We use the notation of $\$ 1$ including the assumptions that $K_{1}$ and $K_{2}$ are complexes selected from the list in Proposition 1.1 and that all coefficients are $Z_{2}$. We will prove that $\Phi_{2}^{4}\left(K_{1} \times\right.$ $\left.K_{2}\right) \neq 0$.

Consider first the following commutative diagram in which the rows are the 
exact Mayer Vietoris sequences given by Lemma 1.6:

$$
\begin{array}{rrrrr}
H^{3}\left(J_{1}^{\prime}\right) \oplus H^{3}\left(J_{2}^{\prime}\right) \rightarrow & H^{3}\left(J_{0}^{\prime}\right) & \stackrel{\delta^{\prime *}}{\rightarrow} & H^{4}\left(\Sigma_{2}\left(\mathrm{R}^{3} \times \mathrm{R}^{3}\right)\right) & \rightarrow H^{4}\left(J_{1}^{\prime}\right) \oplus H^{4}\left(J_{2}^{\prime}\right) \\
& F_{0}^{*} \downarrow & & F^{*} \downarrow \\
H^{3}\left(J_{1}\right) \oplus H^{3}\left(J_{2}\right)^{i \dagger+i^{*}} & H^{3}\left(J_{0}\right) & \stackrel{\delta^{*}}{\rightarrow} & H^{4}\left(\Sigma_{2}\left(K_{1} \times K_{2}\right)\right)
\end{array}
$$

Since $H^{j}\left(J_{1}^{\prime}\right) \cong H^{j}\left(j_{2}^{\prime}\right) \cong H^{j}\left(\mathbf{R} P^{2}\right)=0$ for $j>2, \delta^{\prime *}$ is an isomorphism. Since $\Phi_{2}^{4}\left(K_{1} \times K_{2}\right) \neq 0$ if $F^{*} \neq 0$, we have $\Phi_{2}^{4}\left(K_{1} \times K_{2}\right) \neq 0$ if $\operatorname{Im}\left(F_{0}^{*}\right) \not \subset$ $\operatorname{Im}\left(i_{1}^{*}+i_{2}^{*}\right)$.

Now consider the following commutative diagram in which the rows are exact Gysin sequences or sums of Gysin sequences:

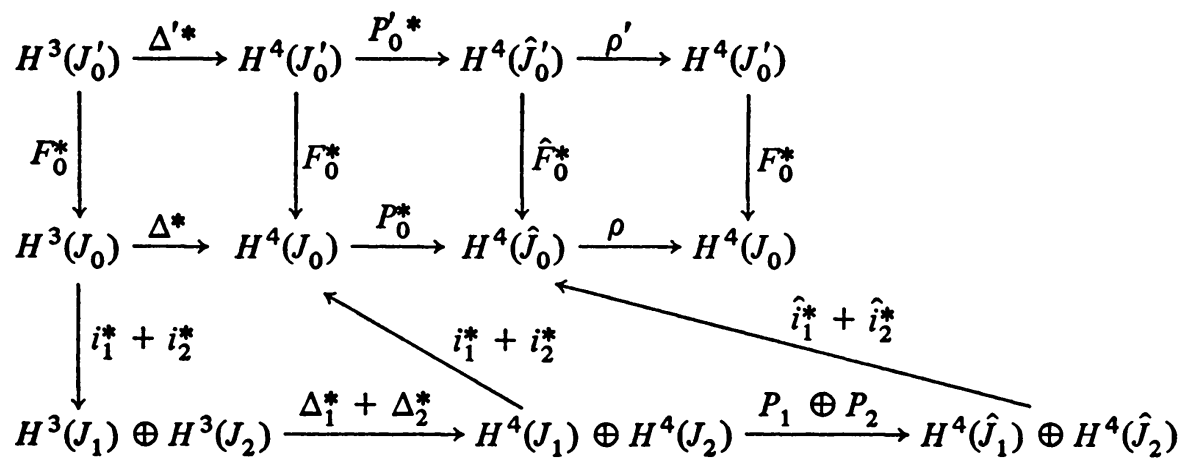

Since $\hat{J}_{0}^{\prime}$ is $\pi$-equivariantly homotopy equivalent to $S^{2} \times S^{2}, H^{4}\left(\hat{J}_{0}^{\prime}\right) \cong$ $H^{4}\left(J_{0}^{\prime}\right) \cong H^{3}\left(J_{0}^{\prime}\right) \cong Z_{2}$ and $\Delta^{\prime *}$ and $\rho^{\prime}$ are isomorphisms. By Proposition 1.3, $H^{4}\left(\hat{J}_{0}\right) \cong H^{4}\left(J_{0}\right) \cong Z_{2}$ and by exactness $\rho$ is an isomorphism. By Lemma 1.7, $F_{0}^{*}: H^{4}\left(J_{0}^{\prime}\right) \rightarrow H^{4}\left(J_{0}\right)$ is an isomorphism. Let $\alpha$ be the nonzero element of $H^{3}\left(J_{0}^{\prime}\right)$. We need to show there do not exist elements $\alpha_{1} \in H^{3}\left(J_{1}\right)$ and $\alpha_{2} \in H^{3}\left(J_{2}\right)$ such that $i_{1}^{*}\left(\alpha_{1}\right)+i_{2}^{*}\left(\alpha_{2}\right)=F_{0}^{*}(\alpha)$. Since $F_{0}^{*}\left(\Delta^{\prime *}(\alpha)\right) \neq 0$, it suffices to show that

$$
i_{1}^{*}\left(\Delta_{1}^{*}\left(\alpha_{1}\right)\right)+i_{2}^{*}\left(\Delta_{2}^{*}\left(\alpha_{2}\right)\right)=0 .
$$

By exactness and symmetry this follows if we prove

$$
\operatorname{ker}\left[p_{1}^{*}: H^{4}\left(J_{1}\right) \rightarrow H^{4}\left(\hat{J}_{1}\right)\right] \subseteq \operatorname{ker}\left[i_{1}^{*}: H^{4}\left(J_{1}\right) \rightarrow H^{4}\left(J_{0}\right)\right] .
$$

This is exactly what was proven in Lemma 2.1. Thus the proof of Theorem $P$ is complete.

\section{REFERENCES}

1. A. H. Copeland, Jr., Deleted products of cones, Proc. Amer. Math. Soc. 16 (1965), 496-502.

2. D. Husemoller, Fibre bundles, Springer-Verlag, Berlin and New York, 1975.

3. S. Mac Lane, Homology, Springer-Verlag, Berlin and New York, 1963.

4. S. Mardesić and J. Segal, On polyhedra embeddable in the 2-sphere, Glasnik Mat. Ser. III 1 (21) (1966), 167-175. 
5. K. Menger, Über plättbare Dreiergraphen und Potenzen nicht plättbarer Graphen, Ergebnisse Math. Kolloq. 2 (1929), 30-31.

6. E. Spanier, Algebraic topology, McGraw-Hill, New York, 1966.

7. B. Ummel, Some examples relating the deleted product to imbeddability, Proc. Amer. Math. Soc. 31 (1972), 307-311.

8. Imbedding classes and n-minimal complexes, Proc. Amer. Math. Soc. 38 (1973), 201-206.

9. W. T. Wu, A theory of imbedding, immersion, and isotopy of polytopes in a euclidean space, Science Press, Peking, 1965.

Department of Mathematics, University of Wisconsin, MilwaUkee, Wisconsin 53201

Current address: Applied Mathematics Group, Energy Technology Applications Division, Boeing Computer Services Company, P.O. Box 24346, Seattle, Washington 98124 